\title{
Formación de precios de los productos de información en redes digitales*
}

\author{
Andrade, Jesús Alberto**
}

\section{Resumen}

Los mercados de redes digitales parecieran señalar el advenimiento de un nuevo paradigma económico, conocido como economía digital. Esta investigación teórica analiza aspectos fundamentales en el desarrollo de una economía de información, la formación de precios de productos de información y su comercialización en los mercados de redes de datos. El análisis demuestra que el término economia digital no conlleva a la creación de un nuevo paradigma económico como su nombre pareciera sugerir, sino que afirma que las leyes económicas de siempre, se aplican por igual a los bienes de información que se venden en los mercados de redes digitales.

Palabras clave: Economía digital, bienes de información, comercio electrónico, precios discriminatorios.

Recibido: 00-03-13 . Aceptado: 00-05-25

* Esta investigación fue realizada en parte gracias al apoyo dado por el Faculty Enrichment Program Award del gobierno Canadiense, durante el periodo académico 1998/1999. Este trabajo es una versión preliminar de un trabajo de investigación en ejecución (No. 1386-99) con fondos del Consejo de Desarrollo Científico de LUZ.

** Economista, Magister en Computación Aplicada y Master in Management Intormation Systems. Profesor Títular de la Licenciatura en Computación de LUZ y de la Maestría en Planificación y Gerencia de la Ciencia y la Tecnología. Departamento de Matemáticas y Computación. Facultad de Ciencias. Universidad del Zulia. Maracaibo, Venezuela. email address: jandrade @iuz.ve. 


\title{
Price Determination for Informational Products in Digital Networks
}

\begin{abstract}
The digital network market seems to point out the creation of a new economic paradigm, known as Digital Economy. This theoretical research analyzes the main issues that influence in the development of an information economy, the price formation of information goods, and their marketing in the data networks. The analysis demonstrates that the concepts of Digital Economy do not generate the creation of a new economic paradigm as this concept seems to suggest; on the contrary, the traditional economic laws apply evenly to all information goods that are sold on the market of digital networks.
\end{abstract}

Key words: Digital economy, information goods, electronic market, discriminatory prices.

\section{Introducción}

Desde los siglos XV y XVI, las sociedades medievales transitaron hacia formas de organización más modernas de las entonces conocidas. Este tránsito se caracterizó por un rompimiento de la demarcación del espacio políticamente rígido, hacia formas de organización territorialmente independientes y soberanas. Esto último condujo a la aparición de naciones-estados sustentadas en un poder central con tendencias absolutistas. Un incipiente sistema capitalista acabó con la estructura económica social medieval. La actividad industrial desplazó a la antigua economía agrícola de consumo, al permitir darle salida a los excedentes a través de un floreciente comercio mundial, cuya gran fuente de riqueza estuvo representada por las colonias.

Hasta el siglo $X X$, las economías mundiales fueron espacios separados, solamente interconectados por medios de transportes lentos y limitados. A comienzos de la segunda mitad del siglo $X X$, los procesos de descolonización reafirmaron la independencia política de las naciones. Mientras que los cambios politicos se han acentuado desde entonces, consolidando la independencia y la soberanía política, la historia de las naciones se ha caracterizado por una expansión económica y comercial sin fronteras. $Y$ a pesar del intercambio económico internacional, las economias de las naciones independientes han sido vistas como un asunto nacional, autónomo y políticamente soberano. El concepto de estado territorial ha dominado el mundo político desde la era feudal, sin embargo, organizaciones no territoriales como las corporaciones multinacionales han controlado económicamente vastos espacios que trascienden las fronteras territoriales. Keohane y Nye (1998), al analizar las po- 
líticas de las transnacionales en el comercio, las relaciones monetarias y las politicas de los espacios comunes afirmaban que el nuevo mundo se solapa y descansa en un mundo tradicional, en el cual los poderes dependen de las instituciones ubicadas en múltiples áreas geográficas; con estados que comandan y gobiernan la confianza de la vasta mayoría de la gente, y que controlan la mayor parte de las riquezas mundiales.

Desde un punto de vista estrictamente económico, el conocimiento adquirido desde el tiempo de aquellas sociedades medievales hasta nuestros días es definido por dos importantes momentos industriales: El primero, la era de la máquina, que surge como consecuencia de la revolución industrial cuando el conocimiento artesanal se organizo y reestructuró para alcanzar una producción mecánica mucho más eficiente. Este primer movimiento se caracteriza por el aumento en la velocidad de producción y en la reducción del tiempo de esa producción, y cuya competencia económica se sustenta en la producción en masa. El segundo, la era de la información, que surge como consecuencia de la revolución social del computador donde los datos pudieron ser almacenados y transformados en información y ésta a su vez en conocimiento para posterior distribución y reuso. Este segundo momento se caracteriza por el amplio espectro que la información alcanza, puesto que es producida socialmente para crear conocimiento.

En ambos casos, la sociedad civil desarrolló habilidades para actuar racional y oportunamente en el mercado, sentando las bases para una nueva economia. Así lo ha argumentado Habermas (citado por Breston, 1997), quien afirma que lo oportuno y lo preciso de la información concerniente al comercio es el sine qua non para un mercado racional y de economía abierta.

De 10 antes expuesto se deduce que se está alcanzando el punto en el cual las naciones se erigen sin fronteras comunicacionales, guiadas por tecnologías que crean sociedades de información globalizadas e interconectadas y, sin embargo, tal globalización está muy lejos de ser universal en cuanto a su alcance territorial y económico. Vivimos en un período histórico de cambios tecnológicos que es inédito en cuanto a su velocidad de adaptación y en cuanto a la modificación de la mayoría de los aspectos económicos, industriales y sociales. No se puede ignorar el poder político y económico que el desarrollo de las nuevas tecnologías de información nos brindan. Se requiere aumentar las capacidades para percibir los efectos que se producen en la sociedad, que son consecuencia de una nueva era influida por los cambios tecnológicos que en ella se producen. Particularmente a partir de los 90, las tecnologías de información han alterado las formas tradicionales de hacer negocios, especialmente en los paises altamente industrializados donde el crecimiento positivo de las variables macro económicas es conducido por el desarrollo de industrias relacionadas con las tecnologías de información.

Las organizaciones más eficientes de hoy en día se ubican en el contexto de la era de la información; cuya competencia se sustenta en un cambio del comercio de productos tangibles a un comercio de productos intangibles, particularmente 
de información. Las redes de datos disponibles en la actualidad han acelerado el reparto y distribución de esos productos de información fuera de las fronteras de las organizaciones $y$ aún fuera de las fronteras de las naciones, sin que se le impongan límites a los desarrollos económicos individuales.

'En los últimos veinte años, el desarrollo tecnológico de las telecomunicaciones y de los componentes electrónicos ha permitido la creación de una nueva forma de desarrollar la economía. Se piensa que ella es el comienzo de un nuevo paradigma económico conocido como economia digital, que en principio refiere a una economía basada en el intercambio de bienes y servicios cuyo desarrollo, producción, venta, y distribución dependen de una tecnología digital. Diversos autores que promueven estas ideas, más que sentar las bases para la creación de un nuevo paradigma económico, preconizan -sin mucha explicación-que estamos en presencia de una nueva economia que dejará atrás la manera como se realiza la actividad económica; y que, además, generará nuevas leyes en las que los viejos conceptos no podrán ser nunca más asociados a la ciencia económica. Tapscott (1996), quien acuñó el término economía digital, provee una diversidad de ejemplos que muestran como las tecnologias de información juegan un papel predominante en el funcionamiento de las actividades de las organizaciones, en el aprendizaje colectivo y en el rol de la Internet en fomentar el comercio electrónico. Sin embargo, no se provee de una conceptualización o referencia teórica que señale qué es la economía digital y cuáles son sus fundamentos.
Este trabajo analiza aspectos fundamentales en el desarrollo de una economía de bienes de información. Prime$\mathrm{ro}$, analiza conceptos relacionados con la formación de precios de bienes de información que suelen ser utilizados indistintamente, pero que tienen diferentes significados, tales como: economía digital, economía en redes digitales y comercio electrónico. Segundo, analiza la formación de precios de productos de información basada en los costos de producción y distribución, en el marco del mecanismo de la discriminación y colocación de precios. Por último, se postulan algunas conclusiones derivadas del análisis de lo que es la economía digital.

\section{Productos de información}

La mayoría de los productos que consumimos son bienes formados por átomos que pueden ser transformados en otros átomos, tales productos son tangibles. Por otro lado, las ideas y los pensamientos, aunque podrían no ser categorizados como bienes, sí pueden ser comercializados como tales. Ejemplo de ello son las patentes y los derechos intelectuales que son expresiones de ideas que se comercializan. Las ideas no tienen una forma específica para materializarse, por lo que se requiere de un soporte que permita contenerlas. El soporte donde se guardan las ideas también se comercializa, y los libros son un buen ejemplo de esto. La información plasmada en un libro está almacenada en un medio físico compuesto por átomos. El libro tal como lo conocemos ahora, forma parte de nuestras vidas desde hace menos de 600 años, y desde entonces su razón de ser sigue 
siendo la misma: el libro sive para almacenar información y es un medio de transporte del conocimiento que perdura en el tiempo (Andrade, 1999). Cuando se tiene un libro, una revista, una cinta grabada con sonidos, o un video, en realidad lo que se tiene son objetos compuestos de átomos, susceptibles de ser transtormados en otros átomos.

Hoy en día, para almacenar una idea no se requiere necesariamente de un objeto físico, ya que la forma como se almacenan los datos en un medio electrónico es por medio de impulsos magnéticos que representan información. De acuerdo con Negroponte (1995), desde un punto de vista estrictamente electróni$c o$, los datos están compuestos de bits de información y no de átomos. Un bit es la unidad mínima de información, y su valor individual puede ser interpretado por uno de los dos siguientes estados: hay información o no hay información. Bits agrupados de una determinada manera pueden representar unidades de información con un sentido mucho más amplio, por lo tanto, un conjunto de bits agrupados provee una mayor cantidad de información que bits considerados individualmente.

El definir qué se entiende por productos de información podría resultar muy amplio y poco preciso, ya que el valor económico de tales productos es independiente de sus aspectos físicos o del recipiente que los contiene. Muchas investigaciones reseñan a la industria de la información como aquella conformada por productos pertenecientes al hardware de los computadores, tales como los equipos electrónicos y todos sus accesorios físicos; otras la refieren como aquellos productos derivados de tecnologias digitales.

Este trabajo se centra en una definición de productos de información más amplia que la representación física de los bienes; y coloca a los productos de la industria del hardware al margen de la investigación, de manera que el análisis se orienta hacia aquellos productos en los que el conocimiento es la base de la producción y la transferencia de productos se logra por intermedio de una tecnología digital.

¿Definiremos a los productos de información como aquellos productos, bienes y servicios que pueden ser transformados en bits de información, y que se pueden transferir por intermedio de una tecnología de información electrónica o digital. De tal forma que un libro, una película, la música, la voz, las imágenes, las transacciones bursátiles, o cualquier producto cuyo contenido puede ser transformado en bits, se considerará como un producto de información. Tales productos se caracterizan por el hecho de que sus valores económicos no dependen de la forma física del bien, sino de la calidad de la información y de la forma como se almacena y transporta, en forma oportuna, para generar conocimiento y producir valor.]

\section{Economía digital}

En la era de la máquina, se consideraba a la economía en función de los costos de producción y de las economías de escala. El valor de los bienes se calculaba en función de los costos y las posibilidades de éstos para generar ingresos. En el pasado, la producción en masa de 
bienes tangibles junto a la disminución de los tiempos de producción, dio origen a una segmentación del mercado que condujo al desarrollo de técnicas de manufactura flexibles. Con el tiempo se desarrollaron modelos de producción en masa a la medida de los consumidores, con ofertas de productos diferenciados en ciertos detalles y comercializados como variaciones de los mismos productos, lo cual produjo una segmentación de los mercados que a su vez generaron nuevas economías de escalas. En el caso de los bienes de información, su distribución a través de sistemas de telegrafía, telefonia, radio y televisión, también ha estado sustentada en las economias de escala; en algunos casos el alcance y control de la distribución han estado sujetos a la intervención de los gobiernos con el fin de evitar eventuales monopolios.

La economía mundial a partir de los noventa se caracteriza por una intensa globalización de los espacios donde se realiza. El comercio de bienes ha trascendido las fronteras naturales. Con el advenimiento de la era digital, la economía ha desarrollado nuevas formas de producción mucho más cercanas al consumidor, que se traducen en productos personalizados, ya que se cuenta con información producida de acuerdo al perfil de cada consumidor. La tecnología electrónica permite a las cadenas comercializadoras de bienes tangibles adaptarse a las nuevas oportunidades que el comercio mundial les brinda. La creación de nuevos segmentos de mercados especializados obliga a las empresas más competitivas a cambiar sus formas de producir y de mercadear. Productos tradicionalmente vendidos in situ, como los libros, los discos, los pasajes aéreos y la venta de acciones en mercados financieros, están siendo comercializados via redes de datos ajustándose a patrones de consumo personalizados. De acuerdo con Rayport y Sviokla (1994) en el mundo de hoy, en el cual existe una sobre capacidad de oferta con escasa demanda, las organizaciones deben responder a los deseos del cliente en lugar de simplemente hacer y vender productos y servicios. Las organizaciones deben establecer relaciones con los clientes sobre la base del aprendizaje de sus necesidades, para proveerlos con los productos adecuados. Se requiere entonces establecer relaciones perdurables con los clientes, basadas en darle al cliente el producto que realmente necesita y requiere. En tal sentido, muchos distribuidores tradicionales están adaptando sus sistemas para comercializar sus productos vía Internet.

A pesar de que hoy en día el volumen de comercialización en los espacios digitales es muy bajo, los proveedores y consumidores encuentran en la red alternativas mucho más variadas que aquellas ofrecidas en los mercados tradicionales. El papel de las tecnologías debe estar dirigido a aprender las conductas de los clientes, a través del almacenamiento de información de eventos, a fin de crear conocimiento para poder estrechar la relación organización-cliente. Cada usuario de los mercados de redes digitales genera información que permite a las empresas diseñar un perfil personal del consumidor. Cuando el comprador visita un determinado sitio de la red, la información que se genera alimenta las bases de datos con su perfil de consumidor, el cual es usado por las empresas para ofrecer pro- 
ductos personalizados. En este sentido, cuando un usuario se conecta a un sitio de venta se crean pequeños archivos ${ }^{1}$ en el computador del usuario (el cliente), con un número único que permite hacer seguimiento personalizado de su particular conducta de comprador.

La era de la información ha permitido el desarrollo de una economía basada en el conocimiento, con una alta velocidad de realización de las negociaciones y con un alcance global de sus actividades, con lo cual se crean nuevas condiciones para el crecimiento económico y social. El conocimiento que se tiene de cada usuario es la base fundamental de una economia mucho más inteligente de lo que hasta hace poco era usual. Las industrias exitosas de estos tiempos dependen más de productos de información, que de sus propias fuentes de materias primas.

La economía se está moviendo de una estructura de trabajo basada en la mano de obra, hacia una estructura de trabajo sustentada en el conocimiento. Las decisiones que se toman en los mercados digitales obedecen más a la calidad de la información que se utiliza para generar conocimiento, que a la cantidad de información disponible. La prontitud en obtener la información determina cuán oportuno es el conocimiento generado, $y$ por lo tanto, su valor en el mercado. La disponibilidad de la información en el momento preciso para tomar decisiones es lo que la hace valiosa. Sin embargo, tener la información pero no dominar el conocimiento requerido para utilizarlo en benefi- cio propio es peor que no disponer de cierta información en particular.

Los parámetros que se utilizan para medir la actividad económica están sujetos a las cantidades y precios de los bienes que se intercambian. Sin embargo, nuestras vidas están motivadas por infinidad de cosas que aunque no tienen un precio monetario, tienen gran valor para nosotros. Los intangibles asociados con las ideas, como el conocimiento, son activos que generan un alto valor por sí mismos; y muchas veces no se intercambian como bienes comerciales, sino como parte del activo total de la organización para el bienestar de la sociedad.

La función social de la información trasciende lo meramente económico. A veces los costos de operación son superiores a las ganancias obtenidas; $\sin \mathrm{em}$ bargo, su permanencia en los mercados digitales es necesaria para afianzar la presencia de productos similares en otros mercados más rentables. Las publicaciones en línea, en ocasiones, no obtienen ganancias en ese tipo de mercado, pero su participación es determinante para mantenerse en otros mercados más tradicionales. Periódicos en línea, como $\mathrm{EI}$ Nacional y El Universal en Venezuela y la vasta mayoria de las publicaciones en línea en los mercados globales, utilizan la publicidad y el patrocinio de empresas para superar los costos de operación. En particular, The Wall Street Journal publicado en la red no acepta patrocinio de empresa alguna, y sólo se mantiene con la publicidad y las subscripciones de 
usuarios que pagan por obtener servicios que no se ofrecen en otros mercados. Aunque el volumen de sus suscriptores vía Internet supera los 150.000 , ese comercio electrónico no genera ganancias.

¿El poder de la información es de tal magnitud que muchas revistas especializadas que son distribuidas en formato $\mathrm{fi}_{-}$ sico, están siendo sustituidas por sus ver-? siones electrónicas. Ello se debe a que los costos de operación y mantenimiento de los artículos en papel son más del doble de los que se incurren con las publicaciones en línea. La tendencia es que las versiones electrónicas desplacen a las impresas, y la razón pareciera estar ajustada a los costos de producción más que a cualquier otra razón de tipo psicológico o humano, tales como la mayor o menor dificultad para ser leida-

\section{El mercado de redes digitales}

La definición más común y sencilla de mercado es aquella que se refiere al sitio donde se compran y se venden productos. Tal idea de mercado resulta hoy muy limitada. Mercado no solamente es el lugar donde se intercambian productos, sino que se alude también a las condiciones generales de producción, distribución y consumo de bienes. En su forma más amplia, la definición de mercado presupone la existencia de mecanismos de formación de precios. En el caso de los bienes de información que se comercializan en redes de datos esa definición resulta todavia muy estrecha, ya que el producto de información en sí mismo posee características propias de un mercado más transparente y de mayor alcance geográfico que el de productos tangibles que se comercializan en áreas físicamente distantes.

Los actores de esta economía de redes concurren al mercado digital con una mejor noción de lo que cada uno les ofrece o demanda. Se crea una red de conocimiento mucho más transparente que favorece tanto a oferentes como a demandantes. La red de datos deja de ser un espacio físico concreto para convertirse en un mercado ideal para el desarrollo de una economia abierta y transparente.

En este sentido, el término red se refiere al conjunto de información que en un espacio abstracto genera conocimiento (Andrade et al, 1999). Los compradores usan la red para determinar quien ofrece el mejor producto al mejor precio. Los proveedores concurren preparados para asumir las condiciones cambiantes del mercado, tratando de adaptarse en la forma más expedita a las preferencias del consumidor. A medida que la información fluye se genera una intensa demanda competitiva basada en los requerimientos del consumidor; una demanda que exige respuestas en menor tiempo que las respuestas exigidas en los mercados tradicionales.

La red sine de mercado al facilitar el intercambio entre productores y consumidores, a la vez que fomenta la desaparición del intermediario como agente del proceso productivo, ya que se acorta la cadena productiva entre el evento, dónde y cuándo se demanda el producto, y el consumidor. Así como los proveedores acuden a los mercados a ofrecer directamente sus productos, los consumidores perciben una mayor transparencia de los mercados de redes digitales y por lo tanto 
actúan directamente en él. En la red, los consumidores adquieren más poder pues poseen más información de precios y calidad de una mayor gama de productos $y$ servicios. Se establece una dinámica entre compradores y proveedores que obliga a disminuir los tiempos de realización de las transacciones y del reparto de los bienes, a medida que se eliminan los intermediarios como agentes del mercado. Los tiempos han disminuido de meses a dias, y en algunos casos han disminuido de horas o minutos a partes de segundos, como en el caso de las transacciones bursátiles donde las operaciones se realizan en tiempo real.

Elofson y Robinson (1998) afiman que la búsqueda de productos altemativos a través de los mercados electrónicos debería beneficiar a los consumidores gracias a una mejor competencia de precios. Sin embargo, la producción de bienes de información en estos mercados digitales involucra un alto poder monopólico en aquellos actores que participan del lado de la oferta de bienes; monopolios que son creados a partir del dominio del conocimiento necesario para producir bienes de información y que buscan recuperar los costos fijos generados al producir la primera unidad. Se establece entonces un mercado de redes de información de oferentes con poder monopólico, y de muchos demandantes interconectados a través de un mercado de redes que comparten las mismas necesidades de información.

Sólo por crear un mercado de redes existe una actividad económica mucho más cercana entre los agentes que allí interactúan, que es independiente de los espacios geográficos que los separan. La actividad económica está guiada por un efecto "red" que amplía la necesidad de información, lo cual hace que el proveedor se estimule en tratar que sus productos sean más accesibles a los consumidores. El productor llega a ofrecer productos complementarios absolutamente gratis, lo que le permite posicionarse monopólicamente en el sector donde actúa. Para el usuario, el efecto en red se comporta en función de cómo otros usan los productos, creando una necesidad de utilizar productos estandarizados de uso colectivo y de aceptación general. En tal sentido, el valor que el usuario da a un bien en particular depende de cómo otros usuarios lo estén usando, y esa es la razón primordial por la que tecnologías abiertas y estandarizadas son exitosas comercialmente. Nadie acude al mercado a comprar un producto que no tenga aceptación universal, ya que sería inútil tener un producto de información que pueda ser utilizado únicarnente por quien lo posee.

Un ejemplo representativo de lo expuesto anteriormente es el de la estrategia seguida por los productos Adobe $y$ Netscape. Adobe Acrobat es una familia de productos que permite crear y abrir archivos que están almacenados en formato $\mathrm{PDF}^{2}$, de amplio uso en la red, ya que posibilita una mejor transferencia de archivos vía electrónica. El Adobe Acrobat sive fundamentalmente para crear y leer 
archivos tipo PDF; la parte del producto que sólo sive para leer archivos PDF es de uso libre, y está disponible gratuitamente en la Internet. La parte necesaria para crear los archivos se vende bajo licencia. La estrategia de Adobe consistió en "amarrar" (lock-in) a un gran número de usuarios, brindándoles la posibilidad de obtener el Acrobat Reader (utilizado para leer archivos PDF) en forma gratuita, para crear una red de necesidades en torno al paquete completo. Similar estrategia siguió Netscape al desarrollar productos estándares para cualquier plataforma de computadores, y distribuyéndolos en redes públicas como la Internet. Yoffie y Cusumano (1999) señalan que la estrategia durante el primer año de los comienzos de Netscape (1995) fue clara, entregar su principal producto (browser) en forma gratuita, mientras se dedicaban a construir un comercio electrónico en sus computadores (servidores) conectados a la WEB. El éxito de su browser fue inmenso, millones de usuarios en todo el mundo se sentian amarrados a la cultura Netscape. Sin embargo, las expectativas en torno al comercio electrónico eran mayores que la realidad. Un seguimiento permanente del mercado señaló que la mayoría de sus ganancias provenian de grandes corporaciones que solicitaban browsers y servidores para sus actividades internas. Sus principales clientes estaban construyendo redes internas al estilo de las redes conectadas a la Internet. Una nueva visión de negocios acerca de to que sería el mercado de sus productos empujó a Netscape a desarrollar tecnologías Intranet para gerencias corporati- vas; Netscape se dedicó a cubrir esa demanda y se centró en el apoyo técnico brindado a sus clientes. La estrategia basada en ofrecer gratuitamente el browser condujo a que grandes corporaciones se amarraran no solo a los productos Netscape, sino a los servicios de asesorías técnicas.

Tradicionalmente, las compañías que son dueñas de sus propias tecnologías han podido generar inmensas ganancias a través de la explotación de segmentos de mercados muy específicos; amarrando a sus clientes, haciéndoles depender de sus tecnologías y creando de esta forma sistemas cerrados dificiles de romper con estilos y culturas propias, tal como lo hizo IBM desde sus comienzos en el mundo de la electrónica. Hoy en dia para que una empresa pueda amarrar a sus clientes es necesario que ofrezca productos que sean desarrollados bajo esquemas de tecnologías abiertas y de libre disponibilidad, y en lo posible, debería desarrollar estrategias que permitan a sus usuarios depender de otros productos y servicios complementarios desarrollados por la misma empresa.

\section{Comercio electrónico}

En la era de la máquina, todo el comercio mundial se hacía con productos tangibles. El comercio se sustentaba en productos compuestos de átomos. Además de las variables asociadas al tipo de mercado, a los costos de operación, y a las ganancias, los átomos contenidos en el producto determinaban su precio. En tal sentido, el precio de los productos co- 
mercializados estaba fijado en gran medida por la cantidad de átomos que esos productos tuvieran.

En la era de la información, particularmente con el desarrollo de la electrónica y las comunicaciones, comienza la comercialización de productos de información que no sólo permite movilizar cantidades de átomos, sino que comercializan cantidades de bits. Esta forma de realizar el comercio a través de bits de información pareciera que llegó para quedarse. Negroponte (1995) sostiene que el cambio de átomos a bits es irrevocable e indetenible. La era de la información electrónica sirve de marco para la realización de transacciones de productos compuestos principalmente de bits. Con el tiempo, las ventas de bienes y servicios en forma electrónica serán la base fundamental de la economía. La Internet es la encargada de transformar, a muy bajo costo, la manera como se ha realizado hasta ahora el comercio; ya que brinda la posibilidad de que las organizaciones publiciten sus productos, promuevan sus ventajas y se comuniquen con sus clientes localizados en diversas partes del mundo, a un costo mucho más bajo que si ocurriera en la forma tradicional. Esa nueva forma de hacer el intercambio de bienes a través de las redes de datos electrónicas es conocida como comercio electrónico.

El comercio electrónico es entendido como la venta y compra directa o indirecta de cualquier tipo de información, productos y servicios por medio de redes de computadores, así como también, el apoyo brindado a cualquier tipo de transacción de negocios sobre una infraestructura digital. Es tal la dimensión del comercio electrónico que no puede ser visto como un simple canal usado para mercadear los productos; ni tampoco puede ser visto como uno nuevo que sustituye o se agrega a los ya existentes minoristas, mayoristas e intermediarios. La audiencia que logra reunir el comercio electrónico supera cualquier otra forma de comercio hasta ahora conocida.

Aunque la Internet pareciera ser la infraestructura adecuada para generar comercio electrónico, no es un requisito para su existencia, pues en lo futuro pareciera que todo comercio electrónico será fusionado con nuevas tecnologías de tipo inteligente, multimedias y TV interactiva. Hoy en día la tecnología y los sistemas disponibles para el desarrollo del comercio electrónico permiten aprender los patrones de conducta de los clientes, gracias a la capacidad de almacenar datos en forma histórica que posibilitan hacer seguimiento de los hábitos de cada uno de los consumidores. Por ejemplo, cuando un usuario de la red utiliza los motores de búsqueda de información de acuerdo a determinadas palabras claves, los sitios o nodos que aparecen ofreciendo sus productos, automáticamente colocan mensajes de publicidad (banners) de acuerdo al patrón de búsqueda que el usuario realiza. Esa información es determinante para que se vaya creando un perfil del consumidor. A medida que se visitan distintos sitios ofrecidos en el mercado electrónico, se va depurando el patrón de consumo del usuario. Si un usuario utiliza palabras claves relacionadas con libros sobre salud, muy posiblemente los avisos que aparezcan en el monitor estarán relacionados con servicios o productos de salud. Mientras más depuradas y específicas sean las palabras utili- 
zadas en la búsqueda de información, más preciso será el patrón generado de ese consumidor. La creación de un mercado, con clientes y consumidores finales cada vez más adaptados al uso de medios electrónicos, es la base del futuro de este comercio personalizado, que se ajusta en forma progresiva a los perfiles particulares de cada consumidor.

Estos sistemas personalizados han dejado de ser herramientas estrictamente técnicas dirigidas al apoyo de la toma de decisiones, para convertirse en sistemas socio-técnicos en los que la dinámica de la organización es reflejada en el comercio electrónico. Las fronteras entre lo que es técnico, y lo que es social ya no están demarcadas, sino que forman un sistema integral con políticas más coherentes que aquellos sistemas ofrecidos en espacios separados que en la era de la máquina dividían lo técnico de lo social.

El éxito del comercio electrónico está basado en ofrecer beneficios, tanto para el comprador como para el vendedor, que sean superiores a los alcanzados en los mercados que usan métodos tradicionales. En los mercados electrónicos se encuentran oferentes que tratan de alcanzar grandes masas de consumidores, colocando a su disposición productos de información y servicios totalmente gratis. Si una empresa, con cierto poder monopólico, logra colocar en el mercado algunos tipos de información, ella podría darle valor a ciertos productos que son ofrecidos en forma gratuita. Pero, ¿qué ventajas ofrece el comercio electrónico si no existe una ganancia monetaria? Los proveedores de información en redes de datos tratan de cubrir sus costos de operación a través de la publicidad. El comercio electrónico está más ajustado a la dinámica de la publicidad, el prestigio y el patrocinio de empresas comerciales, que a cualquier modelo económico. Las empresas utilizan estrategias de mercadeo con el apoyo del comercio electrónico desplegando publicidad, avisos clasificados, proveyendo servicios de internet, actuando como servidores de sitios de otras empresas, proveyendo servicios de diseño de páginas WEB, ventas de archivos y productos de información histórica, y otros tantos servicios que ya comienzan a aparecer.

\section{Estructura de costos de los bienes de información}

El análisis de porqué existen bienes que se ofrecen en las redes de datos, libres de cargos monetarios está más relacionado con los términos del mercadeo, la publicidad y el vínculo psicológico de los clientes con los productos, que con los costos asociados a ellos. Al igual que el conocimiento, los bienes de información tienen para los usuarios un aito valor $y$, sin embargo, muchas veces se ofrecen en los mercados digitales sin precio alguno, ya que su contribución al bienestar de la sociedad, por su naturaleza de intangibilidad, es dificil de medir. En ese sentido, a pesar de estar inmersos en una economía que transfiere valor, generado por la dinámica de los mercados de redes y a pesar de que las nuevas tecnologias de información fomentan el comercio electrónico, la economía digital está sujeta a las tradicionales leyes económicas de siempre. 
Existe la creencia de que la información debería ser gratis, ya que su existencia parte de un proceso de creación intelectual difícil de valorar en términos monetarios, y ello como consecuencia de su intangibilidad. Por otro lado, ciertos bienes de información son considerados bienes públicos, lo que supone un reparto gratuito en la sociedad; tal es el caso de los mensajes políticos, la propaganda, los mensajes religiosos, la publicidad y hasta los pensamientos ideológicos que dependen más del deseo de quien los produce para hacerlos llegar sin costo alguno a los receptores, que de los costos asociados en producirlos.

Desde un punto de vista económi$c o$, las ideas se diferencian de los objetos físicos en que no poseen costos de oportunidad (Pohjola, 1998), ya que no existe la posibilidad de realizar una inversión alternativa cuando se desarrollan las ideas. Sin embargo, el hecho de que las ideas no posean costos de oportunidad, no significa que no se puedan comercializar. Para Ghost (1998) en una economía de conocimiento, analizada en su forma más amplia, es razonable tratar como bienes económicos a todas las formas del saber. Por lo tanto, el conocimiento que se deriva de la información, también es factible de ser utilizado como un bien económico. Sólo aquella información factible de ser comercializada es la que el emisor desea crear y enviar al mercado por un precio determinado. Esta idea de tratar al conocimiento como un producto comercial está asociada a las características que poseen los bienes intangibles de tener altos costos fijos al producir la primera unidad, que deben ser recuperados. Sin embargo, los costos de (re)producir las sub- siguientes unidades del mismo bien son despreciables. $O$ como lo establecen Shapiro y Varian (1997), la información es costosa de producir pero muy barata de reproducir. Para producir la primera unidad del bien de información se requiere ejecutar costos de operación muy altos, incluso algunos costos implícitos que son muy difíciles de estimar. Mientras que para las subsiguientes unidades bastará con hacer las reproducciones nece. sarias y llevarias al mercado, generando costos adicionales que no pasan de ser aquellos costos asociados a la fabricación de los soportes físicos donde se almacena la información.

Si producir la primera unidad de un bien de información resulta muy costoso, y si son casi despreciables los costos variables de reproducir las siguientes unidades, en particular por el uso de la tecnología digital, es factible entonces reproducir altos volúmenes de bienes de información sin generar importantes costos adicionales. Por ello, las economias de escala se ajustan muy bien a la producción de bienes de información, pues mientras más se reproducen los bienes, más bajos serán los costos de producción, en particular si se conoce que estos costos son prácticamente cero. ¿Cómo se justifica entonces el precio a pagar por cada uno de esos bienes de información, si prácticamente lo que se requiere es cubrir los costos para producir la primera unidad del bien?

La producción de bienes de información genera costos irrecuperables (costos hundidos) muy altos. Tales costos no son recuperables si la producción se suspende debido a que son independientes del proceso de fabricación. Si los 
costos unitarios aumentan rápidamente, las empresas tendrán que asignar precios competitivos a fin de que la producción no caiga por debajo de sus capacidades, y esto suele ocurrir cuando las salidas de los productos caen por debajo de su nivel técnico óptimo. En economias de escala, los costos irrecuperables son altamente significativos y las empresas enfrentan el dilema de tratar de recupe. rar los altos costos de la primera unidad producida o bajar los precios a un nivel más competitivo que podria generar una guerra de precios. Si esto ocurriera y se generara una guerra de precios, éstos caerian violentamente sin que las empresas pudieran cubrir ni siquiera sus costos variables asociados a esas unidades producidas, mucho menos para cubrir los costos fijos. Sin embargo, debido a que las economias de escala están intimamente relacionadas con los costos irrecuperables, y en particular si estos costos son demasiado altos, las empresas no desearán salir del mercado y mantendrán las esperanzas de obtener ganancias que les permitieran justificar sus inversiones iniciales. Otra característica importante de los productos de información es que ellos no incurren en costos de reparto, ya que al transferirse por vía digital no se acarrean costos por envío, ni por empaquetamiento, ni costos de fletes y seguros.

La fijación del precio deberá considerar, además de los costos, la posición que tiene el producto en el mercado. El productor deberá ajustar sus precios de forma tal que no incentive la participación de otros productores del mismo bien; ni tampoco que su precio aleje a los consumidores o los incentiven al uso de mecanismos de copia electrónica. En ciertas circunstancias, para el consumidor, compartir un bien de información -copiándolo- es mucho más costoso, que para el productor producir una unidad adicional del bien, por lo cual el consumidor preferirá comprar el producto al precio de mercado.

Las empresas saben que la producción y venta de bienes de información genera una competencia muy alta; por ello, los productores de bienes de información prontamente encuentran que sus productos tienen una alta atracción para que otras empresas entren a competir con bienes similares, sobre todo si esos productos tienen un gran éxito en el mercado. La competencia no está basada en ofrecer un mejor precio, ya que los productores conocen muy bien que con altos costos irrecu perables, caer en una guerra de precios los podría sacar del mercado, por lo cual evitan disminuir sus precios, y se dedican a buscar mecanismos para ganar y mantener segmentos de mercados.

\section{Precios discriminatorios}

Un mercado ideal para el productor, que le permita ofrecer sus productos y obtener la máxima ganancia de cada uno de los posibles compradores, está basado en el deseo que tienen los consumidores de obtener los productos que satisfagan mejor sus necesidades; en tal caso seria deseable establecer precios discriminatorios para cada uno de ellos. Sin embargo, en la práctica es realmente imposible obtener de antemano de cada uno de los consumidores, cuánto esta. 
rian dispuestos a pagar por el bien, pues eso dependerá del valor que cada consumidor le dé a cada producto en un momento determinado.

Debido a esa imposibilidad de determinar a priori lo que cada consumidor estaría dispuesto a pagar, los proveedores de bienes de información desarrollan estrategias y criterios de ventas que en la práctica estén correlacionados con el deseo de obtener esos bienes. De tal forma que distintos consumidores pueden compartir sacrificios a fin de sacar ventajas diferenciadas de los productos que desean obtener. Si un comprador desea un producto cuyo precio es relativamente alto -quizás debido a su escasez- entonces, obtener el producto dependerá del deseo de querer pagar por el bien, aquel precio que el oferente ha establecido. Esta idea, de diferenciar los productos para cobrarle a cada consumidor el máximo de lo que está dispuesto a pagar por el producto, está basada en los trabajos de Pigou, quien en 1920 en su libro The Economics of Welfare distinguió tres tipos de maneras usadas para diferenciar los precios, las que denominó como de primero, segundo y tercer grado. Haciendo una similitud basada en las propuestas de Pigou (1920), este trabajo definirá tres tipos de maneras de personalizar los productos: el primer grado corresponde a la personalización del precio, el segundo al desarrollo de versiones del producto y el tercero al agrupamiento de productos para alcanzar descuentos por volúmenes.

El lograr que cada consumidor pague por un bien el precio más alto posible, se basa en dos características: a) personalizar el precio de acuerdo a las características propias de la población (sectorizando el mercado), definiendo con ello el deseo que cada uno tiene de pagar, y b) definir las capacidades y características intrínsecas de cada producto, para ofrecer versiones que capten de los consumidores los distintos deseos que tienen par obtener el producto. La primera de las características está asociada a variables exógenas al producto ya que depende más del deseo que tiene el consumidor por obtener el producto; mientras que la segunda, está asociada a las variables endógenas del producto, ya que están relacionadas a las caracteristicas propias del bien.

\subsection{Diferenciación de Primer grado: Personalizar el precio}

Este primer grado está asociado a las características diferenciales que tiene la población consumidora. La base de la discriminación del precio está sujeta a valores exógenos al producto, ya que tal discriminación dependerá del valor que cada consumidor le dé al producto, lo cual determinará tanto el deseo de éste por obtenerlo, como el deseo de pagar el precio que el productor solicite.

Debido a la posibilidad que brindan los medios y sistemas digitales de hacer un seguimiento a los consumidores, es posible ofrecer los productos de acuerdo a las propias necesidades y condiciones del usuario. Se crea así, una producción personalizada que se ajusta a las necesidades del cliente. Con este primer grado de diferenciación, los usuarios se deben adaptar a las caracteristicas de la oferta del producto. Un ejemplo de personalizar el precio se da en las librerías en Internet, como Amazon.com o Barnes \& Noble que 
ofrecen en venta libros a precios más económicos que los ofrecidos en sus tiendas y locales, y eso ocurre tan solo por hacer compras via Internet. El patrón de búsqueda que un usuario usa en la Internet le brinda a estas empresas en línea, la posibilidad de obtener del consumidor mucho más información que si la persona comprara en un local, donde paga directamente en caja. La búsqueda de un artículo de acuerdo a un patrón de consumo le da a la empresa información histórica del comportamiento de otros usuarios, por lo que el sistema le permite recomendar otros libros que pudieran ser de utilidad al comprador. Tal recomendación es construida de acuerdo al patrón de consumo de otros clientes que previamente compraron el libro y que se supone se ajusta al patrón de compra del actual consumidor. Las empresas obtienen del consumidor mucho más que dinero por sus ventas, ya que reciben información que convierten en conocimiento para mejorarlas.

Otra forma de personalizar el precio de un servicio o producto de información ocurre cuando se incorporan algunas condiciones especiales de acuerdo al tipo de población, bien sea por su pertenencia a una determinada asociación, o como miembro de una comunidad específica. Por ejemplo, los libros, los dis- cos, los videos en un cierto formato podrían estar dedicados a la población estudiantil, otro formato a los profesores e investigadores, otro a los coleccionistas, etc. Ciertos productos que Microsoft comercializa lo hace bajo el principio de diferenciación de la población. Por ejemplo, el Visual Basic y el Visual $\mathrm{C}++$ se comercializan en dos versiones, una versión estandard para público general y otra más económica dirigida a la comunidad universitaria, conocida como precio académico.

En todo caso, la base de la personalización del producto está orientada por las características poblacionales, por las demográficas, por el poder adquisitivo de los consumidores, y por el valor que cada individuo le da al producto como miembro de una determinada comunidad.

\subsection{Diferenciación de Segundo grado: Versionar el producto}

Si el productor no pudiera diferenciar el producto sobre la base de sus capacidades, no sería posible crear tantos tipos de productos como grupos de consumidores existan con distintos deseos por obtenerlos. En tal caso serían los consumidores quienes terminarían por adaptarse al mercado de esos productos, sin llegar a satisfacer totalmente el deseo

3 El término versionar ha sido introducido por Shapiro y Varian para referirse a las distintas maneras que se utilizan para colocar precios diferentes a los productos de información, sin incurrir en altos costos $u$ ofender a clientes. En la revista Harvard Business Review, noviembre diciembre 1998, aparece un artículo de Shapiro y Varian denominado Versioning: The Smart Way To Sell information, donde señalan distintas formas de versionar productos usando la velocidad, la comunidad, la manipulación y el asombro del público. 
personal por obtener uno por cada producto deseado en particular. En tal sentido, el segundo grado permite que la diferenciación se haga sobre la base de la diversidad de los productos ofrecidos.

Cuando se realizan distintas versiones del mismo producto, se le brinda a los usuarios la oportunidad de adaptarse a las necesidades y a las posibilidades que ellos tienen para obtenerlo de acuerdo a una oferta que el productor hace. La situación ocurre cuando un producto con capacidades bien definidas, colocado con el precio más alto que alguien esté dispuesto a pagar por obtenerlo, puede degradarse para ofrecerlo a un precio menor, de manera de diferenciarlo de aquel producto que se ofrece con todas sus capacidades en plenas condiciones de funcionamiento. Por ejemplo, una música que tenga capacidad sonora en estéreo, puede ser comercializada sobre la base de un precio alto, mientras que el mismo producto se puede degradar con un sonido monoural, para aquellos consumidores que están deseosos de pagar por esa música un precio más bajo. Otro caso con la música, pudiera ocurrir cuando el tamaño del archivo donde se almacenan los bits que representan la música, ocupa una cantidad en memoria mucho menor para quien paga más, y un archivo más grande, digamos tres veces el tamaño del original, para aquellos compradores que no desean pagar el precio más alto. Lo mismo ocurre con fotografías de alta definición que pueden ser degradadas hasta alcanzar una menor resolución para ser vendidas a quien no esté dispuesto a pagar un precio alto.

\subsection{Diferenciación de Tercer grado: Agrupamiento de productos}

En la práctica, el productor prefiere ofrecer descuentos por volúmenes de compra, en lugar de degradar el producto en varias opciones que pudiera significar un engorroso sistema de colocación del producto en el mercado. De tal forma que los productores de bienes de información están más tentados a vender grupos o paquetes de productos de información a más bajos precios que si se vendieran separadamente. El precio bajo por volúmenes está dirigido al consumidor que no desea pagar un precio alto por obtener una sola unidad del producto, y tomará ventaja de los descuentos ofrecidos bien sea de la venta por volúmenes o por paquetes de productos. El consumidor que desea tan solo una unidad deberá entonces pagar un precio más alto por cada unidad individual. En este sentido, la mercadotecnia y la publicidad de los bienes de información inducen al consumo no solicitado, ya que el empaquetamiento de productos ofrece la posibilidad de que el productor agrupe una o varias aplicaciones que no son del interés de un determinado consumidor.

El consumidor obtiene un precio discriminado, que va mejorando a medida que adquiere mayores cantidades de ese particular producto. Tal es el caso de los pequeños inversionistas financieros que, además del precio pagado por una acción, deben pagar los correspondientes gastos por tramitación a un costo realmente alto, a diferencia del gran inversionista que, debido a los altos volúmenes 
de acciones que negocia, sus costos operacionales disminuyen drásticamente, por esta razón, los precios por paquetes accionarios son aprovechados por los grandes inversionistas. La gran mayoría de las empresas que cotizan en las bolsas de valores no ofrecen sus acciones en forma directa a los pequeños compradores que demandan acciones vía Internet, sino que estos compradores deben dirigirse a comprar acciones individuales que fueron obtenidas por empresas de finanzas en paquetes muy grandes.

Idealmente, el productor ofrece al consumidor con mayor deseo por pagar, y que demanda más, una cantidad suficiente para que el deseo de pagar por la unidad adicional sea igual al costo incremental del productor por proveerla. Al consumidor con bajo deseo de pagar por el producto, y por lo tanto con menor demanda, se le ofrece una cantidad ineficiente del bien, por lo que terminará pagando más que el costo incremental por producir la unidad adicional del producto. Para que el agrupamiento de precio ocurra, la oferta al consumidor con bajo deseo por pagar debe ser lo suficientemente ineficiente, para que no atraiga a quien desea y puede pagar más.

Lo difícil de la discriminación de precios es establecer con anterioridad cuántos consumidores desean pagar el precio más alto, para poder determinar con antelación la política de ofertas que deberá seguir. Al ofrecer los productos con discriminación de precios se le da al consumidor la posibilidad de pagar el precio más alto que cada cual está dispuesto a pagar, sin menoscabo de las ganancias del productor.
En la práctica, el productor ofrece combinaciones de productos y servicios por paquetes y versiones, a través del condicionamiento de su uso. Por ejemplo, él pudiera condicionar el tiempo para usarlo, el número de veces que se puede usar, el número de usuarios por producto, y otra gama de posibilidades que se ajustan más a una comercialización basada en la publicidad y la mercadotecnia que en el análisis de costos o en el tipo de mercado en el que se esté comercializando. El agrupamiento es otra modalidad para ofrecer por paquetes versiones de los mismos productos. Muchas veces, productos vendidos individualmente se venden a precios mucho más altos, que si se vendieran en un solo paquete. La razón subyace en el supuesto de que no todo comprador está interesado en obtener todos los productos por su precio original, bien sea por su alto precio individual o porque no representa una verdadera necesidad para el comprador. Si un consumidor individual requiere de alguna aplicación del conjunto de productos ofrecidos en el paquete, se sentirá tentado a adquirir el paquete completo, en caso de una necesidad futura, debido a sus menores costos marginales. Muchos ejemplos se encuentran de tales situaciones. Microsoft comercializa el Office 97 por paquetes de aplicaciones, la versión profesional incluye Power Point, Word, Outlook, Excel y Access; mientras que la versión estandard no incluye el Access. También hay versiones para Windows 95 y para Windows NT. Otra forma de discriminar los precios ocurre dependiendo si el software es almace- 
nado en un CD-ROM o si es almacenado en diskettes, o si es para una comunidad académica o para público profesional.

El productor no tiene por qué preocuparse por los costos asociados en reproducir las siguientes unidades, ya que toda combinación que él haga para lograr el objetivo de vender sus productos, simplemente significará una colocación del producto con bajos (o casi nulos) costos incrementales.

El hecho cierto de que la primera unidad del producto está asociada a altos costos de producción, y las subsiguientes reproducciones poseen costos incrementales despreciables, es lo que motiva al productor a prever un programa de venta basado en la discriminación de precios.

\section{Conclusiones}

La llamada economía digital pareciera ser la expresión económica de una nueva forma de interactuar en los mercados en redes. Sin embargo, esta investigación teórica, sustentada en el análisis de las economias de escala, demuestra que el término economia digital no conlleva a la creación de un nuevo paradigma económico como su nombre pareciera sugerir. Aunque pareciera que estamos en presencia de un nuevo paradigma económico, las leyes económicas de siempre se aplican a los bienes de información que se venden en los mercados de redes digitales, de la misma forma que se aplican a otros tipos de bienes en otros tipos de mercado. Ello es debido a las características muy puntuales que poseen los bienes de información, relacionadas con su capacidad de ser digitalizables, su capacidad de transferencia de datos inmediata, y la de generar altos costos al producir la primera unidad mientras que los costos de (re)producir las siguientes unidades son despreciables. Las siguientes ideas expresan algunas conclusiones que se deducen de este trabajo:

1. Los productos de información que se ofrecen en redes de datos electrónicas pueden ser transformados en bits de información, permitiendo su transferencia por intermedio de tecnologías digitales. Esta facilidad de transferencia es lo que caracteriza al mercado de bienes de información en redes de datos.

2. Los productos de información se caracterizan en que su valor económico dependen principalmente del valor que le otorgue el usuario, de la calidad de la información, y de la forma como se almacena y transporta en forma oportuna. Esa prontitud con que la información se presenta para tomar decisiones es lo que le da valor a la información.

3. La razón del por qué existen bienes que se ofrecen en las redes de datos libres de cargos monetarios, está más relacionada con los términos del mercadeo y la publicidad que con los costos asociados a su producción.

4. El concepto de economía digital, expresada a través del comercio electrónico, no corresponde al nacimiento de un nuevo paradigma económico, sino que el comercio de bienes de información en redes de datos está sujeto a las tradicionales leyes económicas de siempre asociadas a las economías de escala. 
5. Debido a esa imposibilidad de determinar a priori lo que cada consumidor estaría dispuesto a pagar, los proveedores de bienes de información desarrollan estrategias y criterios de ventas que en la práctica estén correlacionados con el deseo que tienen los consumidores de obtener esos bienes.

6. La discriminación de precios se realiza en función de tres tipos de criterios: personalizar el precio, versionar el producto y agrupamiento de productos. A partir de allí los productores generan estrategias de ventas de sus productos.

\section{Bibliografía citada}

Andrade, Jesús (1999). El Hombre del milenio. Diario La Verdad. Maracaibo, Zulia, Venezuela. Pág A5. 10 de enero de 1999.

Andrade, Jesús; Mandrillo, Cósimo, y CampoRedondo, Maria (1999). "La internety el hipertexto en la creación colaborativa de conocimiento". Encuentro Educacional, Vol. 6, 3:261-271.

Breslow, Harris (1997). Civil Society, Political Economy, and the Internet. Capítulo 11. En: Steven Jones (Ed), Virtual Culture Identity \& Communication in Cybersociety. SAGE Publications Ltd. $258 \mathrm{pp}$.

Elofson, G. y Robinson, W.N. (1998). "Creating a Custom Mass-production Channel on the Internet", Communications of the ACM, 41, 3: 56-62.

Ghosh, Pishab Aiyer (1997). Managing First Monday (Managing Editor). Journal on the Internet. Cooking pot markets: an economic model for the trade in free goods and services on the Internet. Disponible en la WWW en la siguiente dirección: http://www.firstmonday,dk/issues/issue3_3/ghosh/in dex.huml. Consulta más reciente: 29 de Febrero de 2000.

Keohane, Robert y Nye, Joseph S. (1998). "Power and Interdependence in the Information Age". Foreign Affairs, Vol. 77, No. 5 (September/October).

Negroponte, Nicholas (1995). Being Digital, Alfred A. Knopf, New York.

Pigou, A.C. (1920). The Economics of Welfare. London: Macmillan.

Pohjola, Matti (1998). Information Technology and Economic Development: An introduction to the Research Issues. The United Nations University. World Institute for Development Economics Research. WIDER. November.

Rayport, Jeffrey F y Sviokla, John (1994), Managing in the market space. Harvard Business Review 1994 Nov; 72(6): 141.

Shapiro Carl y Varian Hal (1997). US Government Information Policy. Trabajo presentado en Highiands Forum del Departamento de Defensa el 8 de junio de 1997. Washington, DC.

Tapscott, Don (1996). The Digital Economy: Promise and Peril In The Age of Networked Intelligence New York: McGraw Hill.

Yoffie, David y Cusumano, Michael (1999) Building a Company on internet Time: Lessons from Netscape. California Management Review. Vol. 41, No 3. Spring. 